\title{
Angiogenesis Inhibitors for Cancer Therapy
}

\author{
Mehdi Rajabi and Shaker A Mousa* \\ Pharmaceutical Research Institute, Albany College of Pharmacy and Health Sciences, USA
}

Received: February 21, 2018; Published: March 01, 2018

*Corresponding author: Shaker A Mousa, The Pharmaceutical Research Institute, Albany College of Pharmacy and Health Sciences, Discovery Drive, Rensselaer, New York, USA, Tel: 518-694-7397; Fax: 518-694-7567; Email: shaker.mousa@acphs.edu

\section{Abstract}

Inhibition of angiogenesis, which refers to blocking the vessel formation from pre-existing blood vessels, has become an attractive target for cancer therapy. Tumors beyond 2-3 $\mathrm{mm}$ in size induce the sprouting of new blood vessels from the surrounding vasculature (sprouting angiogenesis). Design and development of new angiogenesis inhibitors has been validated as a target in several tumor types. In this min-review, we will focus on the role of different anti-angiogenesis strategies and inhibitors in treatment of cancer.

Keywords: VEGF: Vascular Endothelial Growth Factor; TGF: Transforming Growth Factor; TNF: Tumor Necrosis Factor; bFGF: Basic Fibroblast Growth Factor; PLGA: Poly Lactic-Co-Glycolic Acid

\section{Introduction}

Angiogenesis is a complex process in the body. It is the growth of new blood vessels from pre-existing vasculature systems that occur in both healthy and unhealthy blood vessels [1-5]. There are several systemic chemical signals known as angiogenic activators that are involved in repair of damaged blood vessels and induce new blood vessel formation, but on the other hand there is a type of chemical signal known as an angiogenesis inhibitor that induces removal of existing blood vessels. Keeping balance between

angiogenesis inhibitors and angiogenesis activators is important in order to regulate vascular homeostasis (Figure 1) [1]. There are several proteins that control activity of both inhibitors and angiogenesis activators, including platelet-derived endothelial growth factor, vascular endothelial growth factor (VEGF), angiogenin, interleukin-8, hepatocyte growth factor, transforming growth factor $-\alpha$ (TGF)- $\alpha$, TGF- $\beta$, tumor necrosis factor $-\alpha$ (TNF)- $\alpha$, granulocyte colony-stimulating factor, placental growth factor, basic fibroblast growth factor (bFGF, FGF2), and epidermal growth factor.

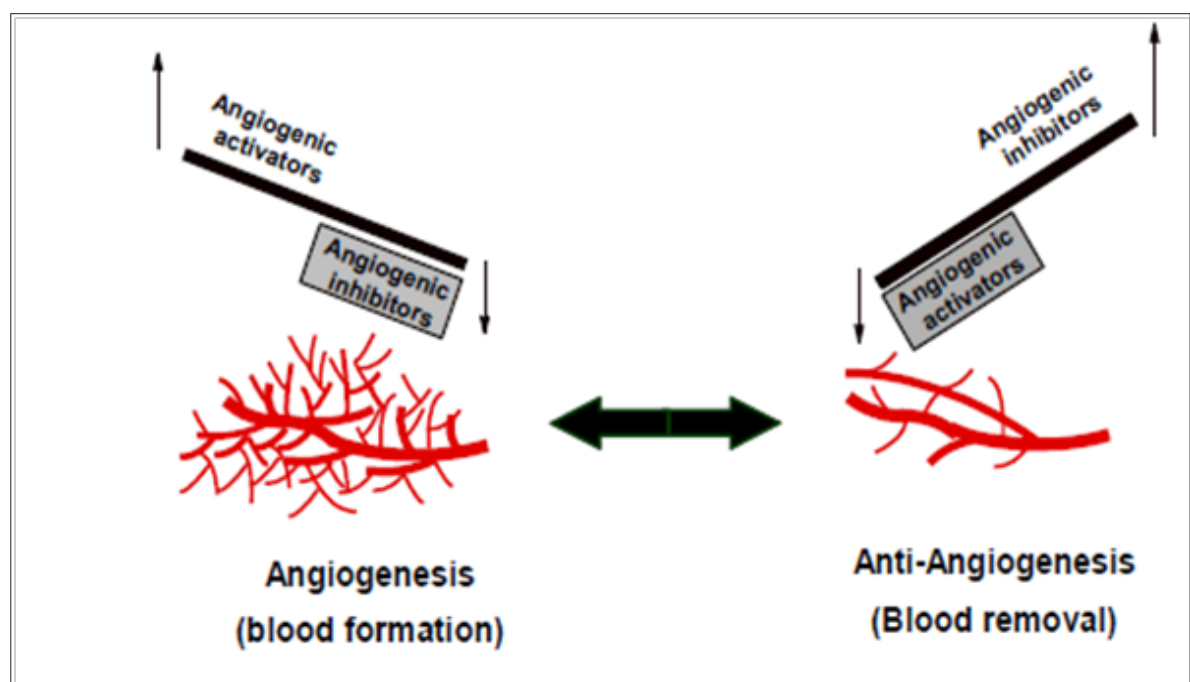

Figure 1: Balance between Angiogenic Inhibitors and Angiogenic Activators in Order to Regulate Vascular Homeostasis. 


\section{Examples of Some Angiogenesis Inhibitors for Cancer Therapy}

There are two main groups for classification of antiangiogenesis molecules: direct inhibitors and indirect inhibitors. Direct inhibitors such as angiostatin, endostatin, arrestin, canstatin, and tumstatin target endothelial cells in the growing vasculature. Indirect inhibitors block the expression or activity of pro-angiogenic proteins like EGFR. In Table 1 are listed some approved U.S. FDA

Table 1: Examples of Some FDA-Approved Inhibitors. angiogenesis inhibitors for the treatment of cancers. Following our interest in searching for new naturally occurring angiogenesis inhibitors, particularly non-protein stimulators of angiogenesis, we previously found that thyroid hormone analogs showed promising pro-angiogenic activity by multiple mechanisms. We reported that tetraiodothyroacetic acid (tetrac), which is a deaminated analogue of L-thyroxine (T4), is able to inhibit the pro-angiogenesis actions of T4 and 3, 5, 3'-triiodo-L-thyronine (T3).

\begin{tabular}{|c|c|}
\hline Generic Name & FDA-approved Indication \\
\hline \multirow[t]{2}{*}{ Everolimus } & Kidney cancer, advanced breast cancer, pancreatic neuroendocrine \\
\hline & tumors (PNETs), and sub-ependymal giant cell astrocytoma \\
\hline Vandetanib, & Thyroid cancer \\
\hline \multicolumn{2}{|l|}{ Cabozantinib } \\
\hline Bevacizumab & Colorectal, non-small-cell lung, and glioblastoma multiforme \\
\hline Thalidomide & Myeloma \\
\hline Lenalidomide & Myeloma (myelodysplastic syndrome (MDS)) \\
\hline Ramucirumab & Stomach cancer and gastroesophageal junction adenocarcinoma \\
\hline Regorafenib & Colorectal cancer and gastrointestinal stromal tumor \\
\hline Ziv-aflibercept & Colorectal cancer \\
\hline Sorafenib & Renal cell and hepatocellular carcinoma \\
\hline Sunitinib & Renal cell and gastrointestinal carcinoma \\
\hline Temsirolimus and & Renal cell carcinoma \\
\hline \multicolumn{2}{|l|}{ Axitinib } \\
\hline \multirow[t]{2}{*}{ Pazopanib } & Renal cell carcinoma, kidney cancer, and advanced soft tissue \\
\hline & sarcoma \\
\hline
\end{tabular}

Tetrac also has high affinity for the integrin - $\alpha \mathrm{v} \beta 3$ receptor, which is important for its interactions with extracellular matrix proteins and other growth factors. Due to huge application of nanotechnology in different biomedicine fields [6-9], and in order to limit the action of tetrac to the integrin $-\alpha v \beta 3$ receptor, we conjugated it to poly(lactic-co-glycolic acid) (PLGA) from its outer ring hydroxyl without any change on the carboxylic acid group in the inner ring [10-12]. We also presented a new pro-angiogenesis modulator called MR- 49 by deiodination of tetrac, which showed significant pro-angiogenic rather than anti-angiogenic activity of tetrac (Figure 2) [13].

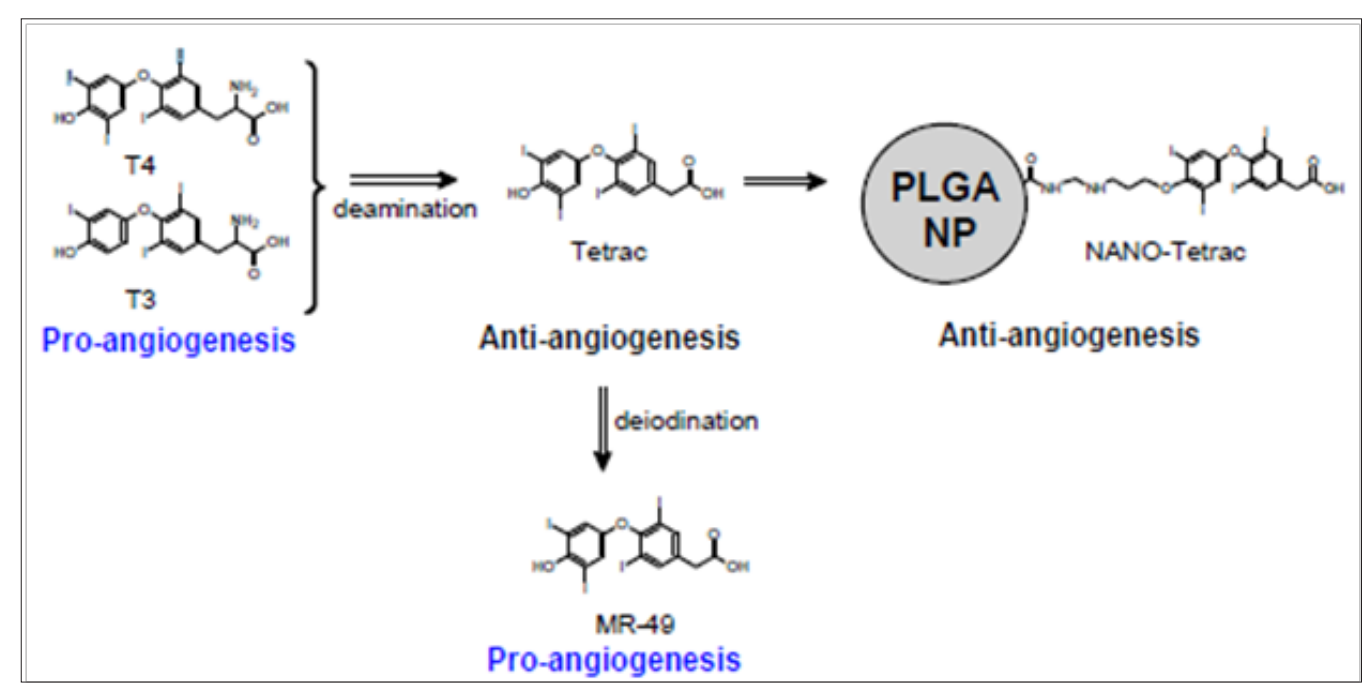

Figure 2: Chemical Structure of Thyroid Hormone Analogs. 


\section{Conclusion}

Angiogenesis, the formation of new blood vessels, plays a significant role in tumor development and might be arrested by effective inhibition of tumor angiogenesis and thereby the growth of tumors may be decreased or stopped. There are two main way including direct or indirect method for designing new angiogenesis inhibitors and sometimes the combination of this anti-angiogenesis agent with some chemotherapy agents, might be essential for effective management of tumor.

\section{References}

1. Rajabi M, SA Mousa (2017) The Role of Angiogenesis in Cancer Treatment. Biomedicines 5(2): 34 .

2. Fuso Nerini, Marta Cesca, Francesca Bizzaro, Raffaella Giavazzi (2016) Combination therapy in cancer: effects of angiogenesis inhibitors on drug pharmacokinetics and pharmacodynamics. Chin J Cancer 35(1): p. 61.

3. Ellis PM (2016) Anti-angiogenesis in Personalized Therapy of Lung Cancer. Adv Exp Med Biol 893: 91-126.

4. Muz B, de la Puente P, Azab F, Azab AK (2015) The role of hypoxia in cancer progression, angiogenesis, metastasis, and resistance to therapy. Hypoxia (Auckl) 3: 83-92.

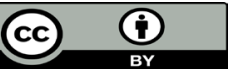

This work is licensed under Creative Commons Attribution 4.0 License

Submission Link: http://biomedres.us/submit-manuscript.php
5. Zhu S, Kisiel W, Lu YJ, Petersen LC, Ndungu JM, et al. (2014) Tumor angiogenesis therapy using targeted delivery of Paclitaxel to the vasculature of breast cancer metastases. J Drug Deliv p. 865732.

6. Rajabi M, SA Mousa (2016) Lipid Nanoparticles and their Application in Nanomedicine. Curr Pharm Biotechnol 17(8): 662-672.

7. Shu Y, Pi F, Sharma A, Rajabi M, Haque F, et al. (2014) Stable RNA nanoparticles as potential new generation drugs for cancer therapy. Adv Drug Deliv Rev 66: 74-89.

8. Shu Y, Yin H, Rajabi M, Li H, Vieweger M, et al. (2018) RNA-based micelles: A novel platform for chemotherapeutic drug loading and delivery. J Control Release.

9. Srinivasan, MM Rajabi, SA Mousa (2015) Multifunctional Nanomaterials and Their Applications in Drug Delivery and Cancer Therapy. Nanomaterials (Basel) 5(4): 1690-1703.

10. Mathangi Srinivasan MR, Shaker A Mousa, Mehdi Rajabi (2016) Chapter 3 - Nanobiomaterials in cancer therapy. 2016(7): 57-89.

11. Mehdi Rajabi MS, Shaker A Mousa (2016) Chapter 1 - Nanobiomaterials in drug delivery. 2016(9): 1-37.

12. Shaker Mousa, Rajabi Mehdi (2017) Non-cleavable polymer conjugated with alpha $v$ beta 3 integrin thyroid antagonists.

13. Rajabi M, Sudha T, Darwish NH, Davis PJ, Mousa SA, et al. (2016) Synthesis of MR-49, a deiodinated analog of tetraiodothyroacetic acid (tetrac), as a novel pro-angiogenesis modulator. Bioorg Med Chem Lett 26(16): 4112-4116.

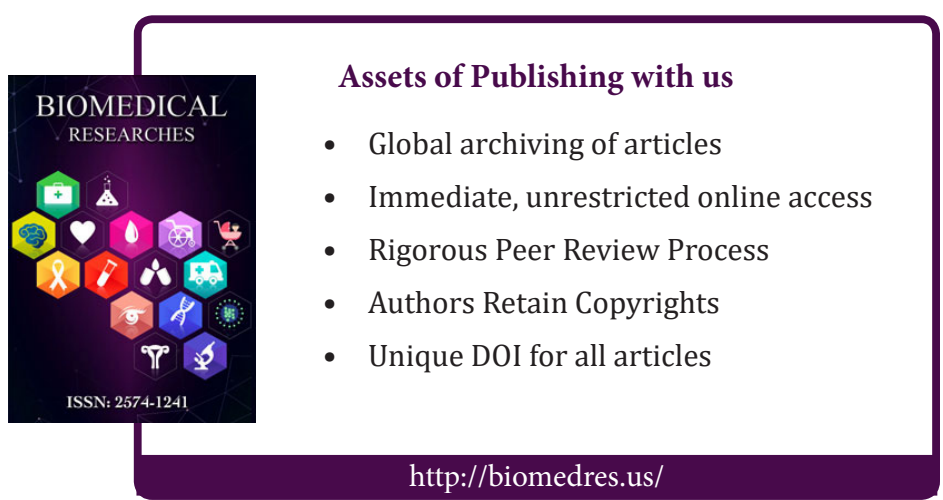

